\title{
In the Wind of Change: Wie Veränderungen im Coaching angestoßen werden können
}

\section{Anhaltspunkte aus Coaching(trainings)gesprächen und deren Nachbesprechungen}

\author{
Sabine Jautz ${ }^{1} \mathbb{D}$ \\ Online publiziert: 9. März 2018 \\ ○ Der/die Autor(en) 2018. Dieser Artikel ist eine Open-Access-Publikation.
}

\section{Zusammenfassung}

Im vorliegenden Beitrag soll gezeigt werden, dass und auf welche Weise das Stellen von Fragen und das Verwenden von Schlüsselbegriffen in Coachinggesprächen zu einem veränderten Sprechen über eine Situation bzw. ein Problem und damit zu Veränderungen in der Einschätzung und/oder Perspektivierung von Klient/innen führen kann. Das wiederum kann die Grundlage für mittel- und langfristige kognitive und/oder Verhaltensänderungen bilden. Hierzu werden diese von Klientinnen und Klienten in Nachbesprechungen als hilfreich bewerteten sprachlichen Mittel mit ihrem konkreten Einsatz in Kurzzeitcoachings abgeglichen. Transkripte von zwei im Rahmen von Weiterbildungen aufgenommen Gesprächen sowie deren Nachbesprechungen sind Gegenstand einer gesprächslinguistisch fundierten qualitativen Analyse. Beim Einsatz von Fragen ist im Verlauf des ersten analysierten Fallbeispiels eine Eskalation zu konstatieren, die sich sowohl formal- als auch funktionallinguistisch manifestiert. Fragen werden dadurch zu einer für den Gesprächstyp spezifischen sozialen Praktik, die der Klientin zu einem Perspektivwechsel verhilft. Ein im zweiten Fallbeispiel zur Zusammenfassung einer Problemlage eingesetzter Schlüsselbegriff ermöglicht dem Klienten eine neue Interpretation seiner Situation. Der Klient greift den von der Coach eingebrachten Ausdruck in seinen eigenen Äußerungen in unterschiedlichen Formen wieder auf und kann, nachdem das Problem erst einmal auf den Punkt gebracht ist, im weiteren Verlauf des Coachinggesprächs gemeinsam mit der Coach konstruktiv Strategien zur Verbesserung seiner Situation entwickeln. Die Arbeit mit Transkripten ermöglicht es, aufzuzeigen, wie sprachliche Mittel in Gesprächen eingesetzt werden und wie ihr Einsatz sich im weiteren Verlauf der Gespräche auswirkt. Eine Sensibilisierung für sprachliche Mittel und Strategien sowie deren Potenziale und Grenzen kann für angehende Coaches in Aus- und Weiterbildung ebenso gewinnbringend sein wie für erfahrene Coaches.

Schlüsselwörter Veränderung $\cdot$ Coachinggespräch $\cdot$ Nachbesprechung $\cdot$ Fragen $\cdot$ Schlüsselwörter

Publisher's Note Springer Nature remains neutral with regard to jurisdictional claims in published maps and institutional affiliations.

Sabine Jautz

jautz@anglistik.uni-siegen.de

1 Seminar für Anglistik, Universität Siegen, Adolf-Reichwein-Str. 2, 57068 Siegen, Deutschland 


\title{
In the Wind of Change: How Changes Can Be Initiated in Coaching
}

Evidence from (Training) Coaching Conversations and their Debriefings

\begin{abstract}
The aim of this paper is to point out that and how asking questions and employing keywords in coaching conversations may result in changed ways of speaking of a situation/problem, which may lead to changes in evaluation and perspectivization. This, in turn, can ultimately be a basis for changes in recognition and behaviour. Questions and keywords have been rated helpful by clients. What they say about the use of these linguistic means in debriefings is compared to the actual use in the coachings preceding the debriefings. Coaching conversations have been recorded in further education settings and then transcribed to allow for a qualitative gesprächs-analytic examination. The questions employed in one conversation escalate formally as well as regarding their communicative functions. In this conversation, asking questions can be shown to be a social practice specific for coaching which helps the client to develop a new perspective. In the second conversation, the coach uses a keyword to summarise what she considers to be the problem for the client. This keyword helps the client to interpret his situation differently. He takes up the keyword in his line of argumentation and once it is clear to him what the problem actually is, he is able to develop strategies to improve his situation together with the coach. Working with transcripts makes it possible to show how exactly linguistic means are employed and which effects they have in the course of conversations. This may prove beneficial to sensitize novice and expert coaches alike concerning linguistic means and strategies, their potentials as well as their limits.
\end{abstract}

Keywords Change $\cdot$ Coaching conversation · Debriefing $\cdot$ Questions $\cdot$ Keywords

\section{Einleitung: Coaching als Ort für Perspektivwechsel}

Im Coaching gibt es verschiedene Möglichkeiten, mit denen Coaches aufgrund ihrer Initiativ- und Kontrollkompetenz in diesem interaktiven professionellen Gesprächstyp (vgl. Graf 2015, S. 3 ff.; Habscheid 2015, S. 263 ff.; vgl. auch Spranz-Fogasy 2014 zu Arzt-Patienten-Interaktionen) Perspektivveränderungen initiieren und gemeinsam mit ihren Klientinnen und Klienten entwickeln können, um diesen zu helfen, ein bestimmtes persönliches oder berufliches Ziel zu erreichen. Sprachliche Möglichkeiten der Initiierung solcher Veränderungsprozesse sollen im Folgenden anhand zweier Fallbeispiele näher in den Blick genommen werden.

Coaching wird verstanden als ,eine zielorientierte, systematische und zeitlich begrenzte Unterstützung eines Entwicklungs- und Veränderungsprozesses im Rahmen beruflicher oder privater Neuorientierung [...]. Übergeordnetes Ziel ist die methodisch geförderte Verbesserung oder Wiederherstellung von professioneller Leistungsfähigkeit und privater Zufriedenheit des Coachingpartners, mit dem Gefühl der autonomen Handlungssteuerung und in Form individuellen Wohlbefindens“ (Nazarkiewicz und Krämer 2012, S. 59). Hierbei spielt Sprache die zentrale Rolle. Sie ist nicht nur Gegenstand der Problemschilderungen, sondern auch das entscheidende Werkzeug, das zur Lösung der geschilderten Probleme eingesetzt werden kann (vgl. Habscheid 2015, S. 256f.). Coaching als Gesprächstyp helfender Interaktion (vgl. Graf et al. 2014, S. 1 ff.) funktioniert dabei als sequentiell organisiertes Gespräch zwischen Interakti- onspartnern mit unterschiedlichen Beteiligungsrechten und -pflichten, Wissensasymmetrien und Perspektivendivergenzen (vgl. Jautz 2017, S. 49 ff.; Nothdurft 1984, S. $67 \mathrm{ff}$.; Nothdurft et al. 1994, darin insbesondere S. 90 ff.). ${ }^{1}$

In der Literatur wird eine ganze Reihe von sprachlichen Mitteln genannt, mit deren Hilfe neue Sichtweisen für Klientinnen und Klienten gefördert und auch hinterfragt werden können. Aus der Vielzahl dieser sprachlichen Mittel, deren Fundus beliebig erweiterbar zu sein scheint, ${ }^{2}$ sollen im Folgenden zwei herausgegriffen werden. Zum einen handelt es sich um das (vielzitierte) Stellen (verschiedener Arten) von Fragen, das der Erfolgsgarant jedes Coachings zu sein scheint (ohne dass dies empirisch begründet würde - vgl. aber Graf und Spranz-Fogasy 2018). Zum anderen sollen Ausdrücke in den Blick genommen werden, die zu Schlüsselbegriffen werden, weil sie Situationen (bzw. ,Probleme‘) oder Strategien (bzw. ,Lösungen“) zusammenfassen und konzise auf den Punkt bringen. Anhand von zwei konkreten Gesprächsbeispielen soll empirisch untersucht werden, inwiefern der Einsatz der genannten Mittel zu verändertem Sprechen über die jeweilige Situation bzw. ,das Problem‘ (vgl. Nothdurft 1984) führt.

In einer Fallstudie beobachten Voutilainen et al. (2011, S. $351 \mathrm{ff}$.), dass sich in den von ihnen untersuchten psy-

\footnotetext{
${ }^{1}$ Im Folgenden soll es primär um Beteiligungsvoraussetzungen gehen.

2 Vgl. Quantifizierungen in Titeln von auf dem Buchmarkt erfolgreichen Publikationen wie. z. B. Wehrle $(2011,2012)$.
} 
chotherapeutischen Gesprächen ${ }^{3}$ Veränderung grob in drei Phasen unterteilen lässt: Zunächst lehnt die Patientin die Schlussfolgerungen ihrer Therapeutin ab, dann reagiert sie ambivalent darauf, bevor sie den Schlussfolgerungen schließlich zustimmt. ${ }^{4}$ Buchholz (2003, S. 90) spricht, ebenfalls mit Blick auf psychotherapeutische Gespräche, in diesem Zusammenhang von ,Umwandlung': Psychotherapie könne nicht nur als ,Problemlösung ' beschrieben werden, ,professionelles Handeln und Behandeln ist weit mehr, nämlich Umwandlung - so, dass aus dem Hilfe Verweigernden ein sein Problem und Hilfe dafür Akzeptierender wird" (Hervorhebung im Original). ${ }^{5}$

Manifestiert sich eine solche Veränderung oder Umwandlung sprachlich, kann dies als Evidenz gewertet werden, dass sich die Klientin oder der Klient innerhalb ihrer/ seiner therapeutic zone of proximal development weiterbewegt. Ribeiro et al. (2013, S. 297) definieren diese Zone als ,the space between the client's current capacity to accommodate the IMs [,innovative moments', ${ }^{6}$ SJ] [...] and a potential capacity that can be reached in collaboration with the therapist". Mit Verweis auf Leiman und Stiles (2001) betonen sie weiter, es gehe hier um eine ,joint activity, not a capacity of client [sic], nor a variable reflecting stable characteristics of the dyad" (Hervorhebung im Original). Damit es tatsächlich $\mathrm{zu}$ Veränderung(en) kommt, ist es also wichtig, dass Coach und Klient/in ihre Beziehung gemeinsam (weiter-)entwickeln und gestalten (vgl. Graf 2015 und Ribeiro et al. 2013, S. $296 \mathrm{ff}$.). Dies stellt laut Konerding (2015) ein entscheidendes Merkmal der ,therapeutischen Allianz' zwischen den Beteiligten dar, die insbesondere auf Affiliation und Alignment fußt, also einem ,willentlichen Verfolgen und kooperativen Bearbeiten eines geteilten Zieles“ bzw. einer „emotionale[n] Konsonanz und vertrauensgetragene[n] Beziehung und Bindung zwischen Kooperationspartnern" (Konerding 2015, S. 273). Im Folgenden sollen - neben Aspekten der Beziehungsgestaltung zwischen Coach und Klient/in verschiedene Praktiken der kommunikativen Basisaktivität

\footnotetext{
3 Wo Forschungsergebnisse aus der jungen linguistischen Coachingforschung noch ausstehen, wird im Folgenden stellenweise auf Forschungsergebnisse aus der etablierten Forschung zu Therapiegesprächen und zu Arzt-Patienten-Kommunikation zurückgegriffen, sofern diese übertragbar sind (vgl. Graf et al. 2014 zu Gemeinsamkeiten und Unterschieden).

${ }^{4}$ Zwischenstufen können hier aus Platzgründen nicht thematisiert werden.

5 Buchholz' (2003) Ausführungen zu Metaphern, in deren Kontext er Umwandlung thematisiert, können hier aus Gründen der Fokussierung ebenso wenig thematisiert werden wie der Prozess als solcher, der in Buchholz und Reich (2015) als „Tanz der Einsicht“ skizziert wird.

${ }^{6}$ Ribeiro et al. (2013, S. 296) definieren diese innovative moments als ,instances in which non-dominant voices express themselves, constitute exceptions to the dominant self-narrative" (Hervorhebungen im Original).
}

„Ko-Konstruieren von Veränderung' (vgl. Graf 2015, in Vorbereitung; sowie Habscheid 2016 zu Praktiken) in den Blick genommen werden. Nach einer kurzen Erläuterung des methodologischen Vorgehens soll dafür die Analyse konkreter Beispiele im Mittelpunkt des Beitrags stehen.

\section{Methodologie: Abgleich von Äußerungen aus Coachinggesprächen und Nachbesprechungen}

Der Untersuchung liegt ein Datenkorpus mit 6,5 Stunden Audioaufnahmen von (voneinander unabhängigen) Coachingsitzungen und sich daran anschließenden Nachbesprechungen zugrunde. Die Daten sind im Rahmen von Weiterbildungen für Coaching und Kollegiale Beratung an Hochschulen sowie für Coaching in interkulturellen Kontexten erhoben worden. Das Datenmaterial enthält (neben zwei Kollegialen Fallberatungen) 13 Kurzzeitcoachings, bei denen die Teilnehmenden der Weiterbildungen in durchschnittlich etwa 20 Minuten zuvor theoretisch Präsentiertes bzw. Erarbeitetes praktisch einüben und anschließend nachbesprechen. Aufgrund des Weiterbildungssettings ist bei den dyadischen Gesprächen häufig noch eine weitere Person anwesend, die bei den Nachbesprechungen eine zusätzliche (Beobachter-)Perspektive einbringt. Auch wenn es sich bei allen Beteiligten um Teilnehmende der Weiterbildungen handelt, sind „echte“ Anliegen derjenigen Teilnehmenden Gegenstand der Coachings, die die Klient/ innenrolle übernehmen. Die Transkription des Datenmaterials erfolgte nach GAT2-Konventionen (vgl. Selting et al. 2009).

Auf der Suche nach Anzeichen für Veränderung wurden, Graf et al. (2010, S. 137) folgend, unterschiedliche methodologische Ansätze interdisziplinär kombiniert: Ethnomethodologische Konversationsanalyse und Kritische Diskursanalyse wurden, wo sinnvoll, ebenso in Anschlag gebracht wie soziolinguistische und pragmatische Ansätze (vgl. auch Kallmeyer 2000, S. 229f. und zum Rückgriff auf die Funktionale Pragmatik insbesondere Scarvaglieri 2015, S. $56 \mathrm{ff}$.). In einem ersten Schritt wurde ermittelt, welche Interventionen insbesondere die Klientinnen und Klienten, aber auch die Coaches und die Beobachter/innen in den Nachbesprechungen als förderlich oder hilfreich für das Erreichen des vorab ausgehandelten Ziels genannt haben, und welche Begründungen sie dafür gegeben haben. In diesen Nachbesprechungen haben Coaches auch verschiedentlich berichtet, welche Interventionen sie in Betracht gezogen haben und warum sie sich schließlich für oder gegen diese oder jene entschieden haben. In einem zweiten Schritt wurden relevante Sequenzen der Coachinggespräche dann mikroanalytisch auf die genannten Aspekte hin untersucht. Für die Analyse besonders rele- 
vant sind die Erwiderungen/Antworten der Klientinnen und Klienten auf Interventionen der Coaches, Wiederholungen von (Schlüssel-)Begriffen durch beide und allgemein (Wieder-)Aufnahmen von Aspekten, die der/die Coach eingebracht hat, in den Äußerungen der Klient/innen (sei es durch Wiederholungen oder durch eine vertiefte inhaltliche Auseinandersetzung mit den angesprochenen Themen). Allgemein ist eine solche Vorgehensweise geeignet, um Good-Practice-Beispiele $^{7}$ zu ermitteln. Das Datenkorpus der hier vorliegenden Untersuchung könnte dafür aufgrund seiner geringen Größe allerdings nur komplementär mit anderen Daten herangezogen werden. Des Weiteren ist zu beachten, dass das Datenmaterial aus Trainingskontexten stammt und damit für diesen Kontext spezifische Praktiken enthält, die größtenteils, aber sicher nicht in Gänze auf Coachings außerhalb von Trainingskontexten übertragen werden können.

Aus Gründen der Fokussierung stellen für den vorliegenden Beitrag die in Nachbesprechungen als hilfreich genannten Aspekte jeweils den Ausgangspunkt dar, von dem aus die Coachinggespräche genauer in den Blick genommen werden. Anhand des Datenmaterials soll gezeigt werden, dass und wie zum einen das Stellen von Fragen und zum anderen Fokussierungen mittels Schlüsselbegriffen in den Coachings zur Etablierung einer anderen Perspektive auf die geschilderte Situation bzw. das geschilderte Problem beitragen können und damit eine Basis für Veränderung bilden. Da Veränderung als von Coach und Klient/in auf unterschiedliche Weise ko-konstruiert verstanden wird (vgl. Graf 2015, in Vorbereitung; Ribeiro et al. 2013, S. $296 \mathrm{ff}$.) und die ,therapeutische Allianz (vgl. Konerding 2015, S. 273 ff.) als für Veränderung konstitutiv erachtet wird, werden im Folgenden jeweils Interaktionssequenzen aus dem Datenkorpus präsentiert. ${ }^{8}$ Die Beispiele sind dahingehend ausgewählt worden, dass sie für das gesamte Korpus typische Fälle zeigen und dass man an relativ kurzen

\footnotetext{
7 Vgl. hierzu die Beiträge beim 61. Arbeitskreis Angewandte Gesprächsforschung (AAG) im November 2017 in Düsseldorf zum Rahmenthema „Die Rolle von ,good practice“ in der Angewandten Gesprächsforschung“.

${ }^{8}$ Graf (2015, S. 5) erläutert die Notwendigkeit der Analyse von Interaktionssequenzen im Zusammenhang mit der Annahme der Ko-Konstruktion von Veränderung näher: ,Da Coaching interaktiv von den Beteiligten ko-konstruiert wird, ist ein einseitiger Fokus auf die Interventionen des Coachs nicht ausreichend, um ihre lokale Funktion und/oder (kommunikative) Wirkung im konkreten Coaching-Gespräch zu bestimmen; stattdessen muss [...] berücksichtigt werden, dass Gesprächsbeiträge sowohl die kommunikative Konsequenz des vorher (vom Gegenüber) Gesagten als auch der kommunikative Ko-Text für das danach vom Gegenüber zu Sagende darstellen. Die jeweilige Bedeutung eines Beitrags wird dabei lokal von den Gesprächsteilnehmer/innen ausgehandelt, das Ergebnis dieser negotiation of meaning (Graf 2007) signalisieren sie sich durch die verbale, non-verbale und paraverbale Gestaltung ihrer Beiträge mit Hilfe von Pausen, Korrekturen, Hörerrückmeldungen etc." (Hervorhebung im Original).
}

Ausschnitten nachvollziehen kann, wie Interventionen der Coaches von ihren Klient/innen aufgegriffen werden. Die Perspektiven der Beteiligten werden dabei (chronologisch und inhaltlich) Schritt für Schritt rekonstruiert.

\section{Analyse zweier Fallbeispiele}

\subsection{Zur Veränderung "durchgefragt": von daher, fand ich das: eh hast dus gut mit (.) mit fragen (-) hinterFRAGT}

Das erste Beispiel entstammt einem knapp zehnminütigen Kurzzeitcoaching mit einer etwa fünfminütigen Nachbesprechung. Drei Teilnehmerinnen der betreffenden Weiterbildung übernehmen die Rollen von Coach, Klientin und Beobachterin. Die Teilnehmerin, die ein persönliches Anliegen zum Gegenstand des Coachings machen möchte, erläutert, dass es ihr nicht gelingt, einen Kollegen davon abzuhalten, ihr wieder und wieder von seinen beruflichen und auch persönlichen Problemen zu erzählen. Im Laufe des Gesprächs erläutert die Klientin, dass sie und ihr Kollege zusammenarbeiten müssen, um ihre jeweiligen Bereiche zu koordinieren. Der Kollege hat zwar einen höheren Status als die Klientin, ist aber nicht ihr Vorgesetzter. Der Gesprächsausschnitt in (1) stammt aus der Nachbesprechung des Coachings, in dem die Klientin das kommunikative Verhalten der Coach kommentiert.

(1) Klientin: e::hm (--) ja: (.) also alles in allem fand ich das: e:hm (--) dann doch ehm (.) doch HILFreich? du hast gut ZUgehört und bis auf: (--) e:h (--) hast mir auch keine lösungs $<<$ leicht lachend $>$ vorschläge $>$ in dem sinne sondern du hast das sehr gut hinterFRAGT. das fand ich GUT ne also- [...] ja? eh $(-)<$ was sagen se DA:zu> und ${ }^{\circ} \mathrm{hh}(-)$ wie jetzt eben zum schluss ne $<$ was wär jetzt das SCHLIMmste-> hh also (.) mich SPREchen lassen. nicht du als experte $<$ ich würde emPFEHlen $>$ sondern, ich bin derjenige der (--) hier sitzt und ich: (.) soll auch was <<leicht lachend $>$ zu dem> thema SAgen. DAS fand ich gut. ${ }^{\circ} \mathrm{hh}$ das hast du gut geLÖST. $h^{\circ}$ hast mich auch zum sprechen geBRACHT (--) das: (.) von daher, fand ich das: eh hast dus gut mit (.) mit fragen (-) hinterFRAGT. (10:06-10:48)

Auf der Grundlage, dass die Klientin Fragen ihrer Coach als hilfreich empfunden hat, soll im Folgenden das eigentliche Coachinggespräch auf solche Fragen und deren Po- 
tenzial, Veränderungen einzuleiten, analysiert werden. ${ }^{9} \mathrm{Da}-$ bei fällt, wie zu zeigen sein wird, eine Eskalierung oder Zuspitzung in den Fragen der Coach auf. Eine Frage ist nicht zuletzt deshalb eine hilfreiche Intervention, weil „sie eine Antwort konditionell relevant setzt“ (Mack et al. 2016, S. 29). Verwendet man sie als Coach im Rahmen der eigenen Initiativkompetenz, liegt der Ball danach im Feld des/der Klient/in. Zum Erreichen des Coachingziels ist er oder sie nun am Zuge, ihn zurückspielen, indem das Erfragte reflektiert wird. Veränderung wird so ko-konstruiert (vgl. Graf 2015, in Vorbereitung).

Im vorliegenden Beispiel handeln Coach und Klientin zu Beginn des Gesprächs kurz aus, was Gegenstand ihres Coachings sein soll und die Klientin beschreibt kurz ihre Situation. Die Coach hört ihr dabei achtsam zu (vgl. Albrecht und Perrin 2013, S. 37 zum aufmerksamen, nondualen und emergenten Zuhören; Menzel 2008), was sie durch viele Rückmeldesignale anzeigt. Die Klientin formuliert schließlich in dem in (2) wiedergegebenen Gesprächsausschnitt ihr Ziel.

(2) Klientin: [ich möchte ihm] DAS vermitteln und möchte [sagen] dass (-) bis HIERhin und nicht WEIter. $(3: 44-3: 48)$

In den folgenden (hier nicht wiedergegeben) Turns des Gesprächs lobt die Coach die Formulierung und fragt die Klientin dann, ob sie ihrem Kollegen das tatsächlich schon einmal so gesagt hat. Die Klientin verneint dies. Das kommunikative Handeln der Coach ist in dieser Phase diagnostisch ausgerichtet (vgl. Graf in Vorbereitung). Sie stellt weitere Fragen dahingehend, ob die Klientin sonst schon etwas ausprobiert habe, um den Kollegen in seinem Erzählen zu stoppen, und wenn ja, was. Die Klientin erwidert, dass sie konkret noch nichts ausprobiert, sondern nur in Gedanken formuliert habe. Die Coach fragt sie, ob sie diese Formulierungen noch einmal wiederholen könne, was die Klientin bejaht. Sie nennt einige Beispiele. Die darauf folgende Frage der Coach ist in (3) wiedergegeben.

(3) Coach: $\mathrm{mhm}{ }^{\circ} \mathrm{hh}(-)$ eh was (.) was vermuten sie (-) wenn sie ihm das so sa:gen was dann pasSIERT? $(5: 49-5: 57)$

Die W-Frage ist offen und im Vergleich zu den noch folgenden (s. unten) neutral formuliert. Sie kann dahingehend interpretiert werden, dass die Coach zunächst versucht, eine Allianz mit der Klientin aufzubauen (vgl. Graf und Pawelc-

\footnotetext{
${ }^{9}$ Fragen als Intervention sind (zumindest im engeren Sinne) erst zu einem späteren Zeitpunkt in der Weiterbildung behandelt worden und waren nicht Teil des theoretischen Inputs, der der Coachingübung vorausgegangen ist.
}

zyk 2014, S. $66 \mathrm{ff}$.) - wie vorab schon mit der positiven Bewertung einer Formulierung und der Fokussierung dessen, was die Klientin schon selbst getan hat, um Abhilfe zu schaffen. Die Klientin erwidert Folgendes:

(4) Klientin: mh::- [...] dass er das zur KENNTnis nimmt und sagt <JA:,> aber beim NÄCHsten mal steht er WIEder da. (5:59-6:04)

Die Klientin könnte aus ihrer Perspektive berichten, geht aber, wie in (4) ersichtlich, nur kurz auf das Angebot/die Frage ein und fokussiert ihrerseits die Aussichtslosigkeit des von der Coach intendierten Vorgehens (,aber beim NÄCHsten mal steht er WIEder da“). Die Klientin lehnt damit zunächst das mithilfe von Fragen durch die Coach entworfene (hypothetische) Szenario ab, dass sie ihrem Kollegen direkt sagt, dass sie sich seine Problemschilderungen nicht länger anhören möchte (vgl. Voutilainen et al. 2011, S. $352 \mathrm{ff}$. zur Phase der Ablehnung als Einleitung von Veränderung in der Psychotherapie). Die Klientin führt im weiteren (hier nicht wiedergegebenen) Verlauf des Gesprächs als Begründung an, dass aufgrund ihrer Zusammenarbeit mit dem Kollegen weitere Treffen unvermeidlich sind - und mit weiteren Treffen sind ihrer Meinung nach auch weitere Problemschilderungen unvermeidlich. Die Coach gibt in der Nachbesprechung an, dass ihr auf Basis der Schilderung der Klientin zunächst nicht klar war, dass die Klientin und der Kollege eng zusammenarbeiten und sich nicht nur zufällig, etwa beim von der Klientin erwähnten Kaffeeholen, treffen und der Kollege der Klientin dann seine Probleme schildert:

\section{(5) Coach: das war ne ganz neue informaTIO:N (13:57)}

Die Coach gibt in der Nachbesprechung an, diese Information im Coaching zum Anlass genommen zu haben, im Folgenden konkreter zu intervenieren und die Klientin, wie in (6) wiedergegeben, nach ihren Befürchtungen zu fragen, was passieren würde, wenn sie ihren Kollegen direkt konfrontierte. Auch hierfür verwendet die Coach wieder eine offene W-Frage:

(6) Coach: das heißt angenommen sie würden ihm das so DEU:Tlich [sa:gen-] wie sie (-) da eben (.) mh (-) haben sie schon den ersten [AN]satz so ge[MACHT, ${ }^{\circ} \mathrm{h}$ was befü:rchten sie was dann pasSIE:RT? (7:04-7:15)

Mit der Verwendung des Verbs ,befürchten' adressiert die Coach negative Gefühle der Klientin (,topicalizing feelings talk“, vgl. Graf und Pawelczyk 2014, S. 72 ff.), die sie möglicherweise davon abhalten, ihren Wunsch gegenüber dem Kollegen offen auszusprechen. ,Befürchten “ ist weniger neutral als das in der vorangegangenen Frage ver- 
wendete Verb ,passieren' (Ausschnitt (3)). Interessant ist, dass die Frage nach den Befürchtungen einerseits durch eine hypothetische Situation gerahmt wird (,,angenommen sie würden ..." unter Verwendung des Konjunktivs) und die negativen Gefühle damit quasi der Realität enthoben werden; dass sie andererseits aber dezidiert mit der Realität des Coachings ${ }^{10}$ verknüpft wird, in der es der Klientin bereits gelungen ist, ihre Wünsche direkt zu formulieren (,wie sie (-) da eben (.) mh (-) haben sie schon den ersten [AN]satz so ge[MACHT,]"). Diese Passage kann hier als positive Bestärkung gelesen werden: Die Klientin hat bereits demonstriert, dass sie ihren Wunsch deutlich artikulieren kann.

Die in (7) wiedergegebene Reaktion der Klientin auf die in (6) zitierte Frage nach den Befürchtungen ist spezifischer und detaillierter als die Reaktion auf die vorangegangene Frage (vgl. (4)).

(7) Klientin: ja dass die weitere zusammenarbeit dann vielleicht nicht mehr GANZ so günstig ist. mit (.) zwischen uns BEIden. (7:16-7:25)

Mit Buchholz (2003, S. 89f.) und Voutilainen et al. (2011, S. $354 \mathrm{ff}$.) ist zu erwägen, ob hier der Beginn einer Umwandlung zu erkennen ist: Nachdem die Klientin zunächst die Aussichtslosigkeit fokussiert hatte (vgl. (4)) und damit die durch Fragen ins Spiel gebrachte Möglichkeit abgelehnt hat, ihren Kollegen direkt zu konfrontieren, lässt sie sich nun darauf ein, über Folgen nachzudenken und ihre Befürchtungen zu verbalisieren. Hiermit verschafft die Klientin einer bislang unterdrückten inneren Stimme Gehör (Albrecht und Perrin 2013, S. 50f. sprechen in einem solchen Fall von nondualem Zuhören) und erlebt damit einen innovative moment im Sinne von Ribeiro et al. (2013, S. 296f.), womit sie (sich) eine Möglichkeit der Weiterentwicklung und Veränderung eröffnet. Die Formulierung „vielleicht nicht mehr GANZ so günstig“ mildert die Beschreibung der Befürchtung aber durch das einschränkende „,vielleicht“, die Relativierung ,nicht mehr GANZ so günstig" und darin durch die Betonung von "GANZ" ab und lässt sie vage erscheinen. In Anlehnung an Voutilainen et al. (2011, S. 354 ff.) kann man hier von einer ambivalenten Erwiderung sprechen.

Die Coach entscheidet sich, bei dieser Ausgangslage nachzuhaken. Sie fragt zunächst nach den Auswirkungen, worauf die Klientin keine konkrete Auskunft geben kann, und fügt dann eine in (8) wiedergegebene elliptische Äußerung mit Frageintonation an, mit der sie die Klientin herausfordert (vgl. Mack et al. 2016, S. 55 f., $72 \mathrm{ff}$.; Ribeiro et al. 2013, S. 298f., 301):

\footnotetext{
${ }^{10}$ Hier ließe sich noch weiter differenzieren, dass es sich um eine ,Coachingrealität im Rahmen einer Coachingweiterbildung ' handelt.
}

(8) Coach: aber ihre schlimmste phantaSIE frau $x$ ? (7:32-7: 36)

In (8) fällt die Verwendung des Superlativs ,,schlimmste" ebenso auf wie die markierte Syntax. Der Einstieg mit „aber“ und die direkte persönliche Adressierung der Klientin mit ihrem Namen haben einen stark auffordernden Charakter, dass die Klientin der Coach und sich selbst einen (überzeugenden) Grund präsentiert, der sie davon abhält, sich zu verbitten, dass der Kollege ihr weiterhin seine Probleme schildert. Das ,,aber“ kann als Einfordern einer Rückversicherung der subjektiven Einschätzung der Klientin gedeutet werden. Man könnte spekulieren, dass es Ungläubigkeit der Coach indiziert und sie eine ,nicht mehr ganz so günstige Zusammenarbeit" selbst womöglich nicht als ,Befürchtung' kategorisieren würde - und durch ihr emergentes, lösungsorientiertes Zuhören gemeinsam mit der Klientin weiterarbeiten möchte, indem sie eine Offenheit für neue Sichtweisen schafft (vgl. Albrecht und Perrin 2013, S. 70f.). Mit der Formulierung „schlimmste phantaSIE“ appelliert die Coach wieder an die Emotionen und an die Vorstellungskraft der Klientin, um die therapeutic zone of proximal development auszuweiten.

Die Klientin scheint auf die implizite Frage nicht direkt antworten zu können. Sie wiederholt in ihrer in (9) zitierten Erwiderung zunächst , $<<$ p $>$ schlimmste phantaSIE, $>$ “ und macht dann eine (Denk-)Pause, bevor sie fortfährt.

(9) Klientin: $<<p>$ schlimmste phantaSIE, $>(2,1)$ ja: dass er vielleicht ehm $(4,5) \mathrm{hh}^{\circ}$ mir gegenüber dann (-) NICHT mehr so: (---) FREUNDlich wäre- ich WEIß es nicht. ich hab (.) ich HAB mirs eigentlich noch nicht über[LEGT was sein könnte;] (7:37-7:53)

Was sie, eingeleitet durch ein langgezogenes ,ja:“, als „schlimmste phantaSIE“ nennt, wird von der Klientin auf vielfache Weise relativiert, angefangen mit ,vielleicht". Es finden sich viele, mitunter sehr lange Pausen (bis zu 4,5 Sekunden lang) und Verzögerungssignale (,ehm“), die Klientin atmet hörbar (,hh ${ }^{\circ}$ “), setzt viele Fokusakzente (,NICHT“, „FREUNDlich“, „WEIß“, „HAB“, „über[LEGT“]) und wiederholt sich (,,ich hab (.) ich HAB“), um schließlich zu sagen, dass sie ,eigentlich" noch gar kein Worst-Case-Szenario entwickelt hat. Die Verwendung des Konjunktivs in „was sein könnte“ lässt das Vorgetragene zusätzlich zu der Relativierung durch „eigentlich“ irreal erscheinen. Die Erwiderung in (9) kann mit Voutilainen et al. (2011, S. $354 \mathrm{ff}$.) insgesamt als ambivalent charakterisiert werden. Das Bemühen, eine (überzeugende) Begründung zu geben, genauso wie das Eingeständnis, aktuell (noch) keine geben zu können, setzt, wie im Folgenden zu zeigen ist, einen Gedankenprozess just dahingehend in Gang, sich darüber klar zu werden, 
ob die genannten Gründe sie (tatsächlich) abhalten, den Kollegen in die Schranken zu weisen. Hierbei haben wir es mit der Entwicklung einer anderen Sichtweise, einer Umwandlung oder Rekonzeptualisierung zu tun (vgl. Buchholz 2003, S. 89 f.; Scarvaglieri 2015, S. 60 ff.). Die Klientin führt in den folgenden (nicht wiedergegebenen) Turns weiter aus, dass der Kollege ihr womöglich künftig seltener Gefälligkeiten erweisen würde (wie etwa, Dokumente für sie zu transportieren), wenn er ihr nicht mehr sein Leid klagen könne.

Die Coach versichert sich im Anschluss mit einer weiteren Rückfrage, dass die Klientin ihre vorangegangene Frage für den Moment abschließend beantwortet hat und betont noch einmal, worum es ihr mit der Frage ging.

(10) Coach: und das ist das was am !SCHLIMM!sten (--) passieren, $(2,0)$ ist das so die größte ist das eh die (.) ist das jetzt die- $(2,0)$ also ich hab so wirklich nach dem gefragt (.) [nach] dem !SCHLIMM!sten (-) [was] pasSIEren könnte- (9:19-9:26)

Die in (10) wiedergegebene Äußerung enthält verschiedene syntaktisch unvollständige Konstruktionen. Zu Beginn finden sich Teile von geschlossenen Entscheidungsfragen (,und das ist das was am !SCHLIMM!sten (--) passieren, $(2,0)$ ist das so die größte ist das eh die (.) ist das jetzt die- $(2,0)^{\prime \prime)}$ bevor die Coach ihre offene W-Frage ,nacherzählt“ (,also ich hab so wirklich nach dem gefragt (.) [nach] dem !SCHLIMM!sten (-) [was] pasSIEren könnte“). Die Äußerung ist durch Pausen und Reparaturen gekennzeichnet. Die Betonung mittels „wirklich“ und Fokusakzenten sowie die Wiederholung von schon Gesagtem (,also ich hab so wirklich nach dem gefragt (.) [nach] dem !SCHLIMM!sten (-) [was] pasSIEren könnte-“) machen deutlich, dass es der Coach um die Schilderung des Worst-Case-Szenarios ging (vgl. auch die zweimalige Verwendung von ,!'SCHLIMM!sten“). Sie spiegeln ferner den Eindruck, dass die Coach der Klientin Gelegenheit geben möchte, den ,schlimmsten Fall' gegebenenfalls neu zu bewerten, der sie nach eigenen Angaben davon abhält, dem Kollegen klar zu sagen, was sie (nicht) möchte. Die Klientin ist aufgefordert bzw. herausgefordert (vgl. Mack et al. 2016, Kapitel 2 und 4; Ribeiro et al. 2013, S. 299), sich ihre eigene Argumentation noch einmal vor Augen zu führen und für das weitere Gespräch als gültig zu ratifizieren - oder zu verändern.

(11) Klientin: [ja:;] [nja::;] ja. ich denke SCHON. also ich denk a je (.) wie gesagt er ist ja nicht mein VORgesetzter (.) also inSO:fern- (9:27-9:38)

In ihrer in (11) gezeigten Erwiderung wägt die Klientin ab (,,[ja:;] [nja::;] ja.“), stimmt vorsichtig zu (,,ich den- ke SCHON.“) und setzt dann zu einer Wiederholung an (,also ich denk"), die dann aber in die Andeutung einer neuen Sichtweise mündet: ,a je (.) wie gesagt er ist ja nicht mein VORgesetzter (.) also inSO:fern-“. Diese Information ist an dieser sequentiellen Position im Gespräch neu. Mit dem nicht weiter ausgeführten ,also inSO:fern“ bleibt das Argument in der Schwebe bzw. die Klientin überlässt die Komplettierung des Arguments der Adressatin. Man kann die Andeutung dahingehend verstehen, dass sie bei einem durch die Artikulation ihres Wunsches gestörten Verhältnisses mit dem Kollegen zumindest keine dienstlichen/dienstrechtlichen Konsequenzen zu befürchten hat, da sie in keinem Abhängigkeitsverhältnis zu ihm steht. Dass dieses rekonstruierte Argument der zuvor geäußerten Ablehnung widerspricht bzw. diese zumindest relativiert, lässt sich auch an der Verwendung von ,a je (.)“ ablesen. Die Klientin bemüht sich aber, das Argument inhaltlich trotzdem in ihre Gesamtargumentation einzubinden (,wie gesagt") und damit ein konsistentes Selbstbild zu konstruieren (vgl. Brown und Levinson 1987, S. 61 ff.). Im Vergleich mit den vorangegangenen Äußerungen der Klientin wirkt es, als sei die Äußerung in ihrer Ambivalenz einer Zustimmung (oder „,resigned acceptance“, Voutilainen et al. 2011, S. 360) näher als einer Ablehnung und als bewege sie sich näher an die obere Grenze der möglichen Entwicklung (vgl. Ribeiro et al. 2013, S. 299). Genaueres lässt sich mit Blick auf dieses Coachinggespräch selbst nicht sagen, da es unmittelbar nach der in (11) zitierten Äußerung zu einer Störung von außen kommt und zudem im Rahmen des Weiterbildungssettings ein Hinweis folgt, dass die für das Coaching vorgesehene Zeit abgelaufen ist.

Coach und Klientin eröffnen im Anschluss daran sofort die Nachbesprechung des Kurzzeitcoachings (vgl. die eingangs in (1) zitierte Äußerung). Hier bestätigt die Klientin noch einmal, dass sie sich über die Konsequenzen einer Artikulation ihres Wunsches vorab noch keine Gedanken gemacht hatte.

(12) Klientin: ja weil ich (-) da ist es mir nämlich erst beWUSST geworden wo (.) [was hab ich jetzt] eigen wo IST eigentlich dein ${ }^{\circ} \mathrm{hh}$ [was WILLST du eigentlich] ne, ist dir das EIne wichtiger oder das ANdere [wich]tiger. was möchteste ne, [das ist] mir aber JETZT erst klar geworden (12:49-12:59)

Die in (12) wiedergegebene Äußerung ist durch viele Abbrüche und Neuansätze gekennzeichnet - die Klientin scheint ihre Gedanken und deren Verbalisierung erst ordnen zu müssen und behilft sich dabei mit an sich selbst gerichteten offenen und geschlossenen Fragen (, ${ }^{\circ}$ hh [was WILLST du eigentlich] ne, ist dir das EIne wichtiger oder das ANdere [wich]tiger. was möchteste ne“). 
Es fehlt eine konkrete Benennung dessen, was die Klientin möchte und was ihr wichtiger ist, aber Äußerungen aus der Nachbesprechung wie die in (12) wiedergegebene deuten auf eine stillschweigende Übereinkunft, dass der beschriebene Worst Case ein Risiko ist, das es sich einzugehen lohnt; dass die Klientin also versuchen könnte, ihrem Kollegen klar zu sagen, dass sie künftig nicht mehr möchte, dass er ihr seine beruflichen und privaten Probleme erzählt. Die Analyse zeigt, dass der Einsatz unterschiedlicher Fragen, die sich in diesem Fall auf ein Worst-Case-Szenario beziehen, ${ }^{11}$ in ihrer Eskalation zur Folge haben, dass sich die Klientin in einer diagnostischen oder explorierenden Phase (vgl. Graf in Vorbereitung) zunächst gedanklich darauf einlässt, zu überlegen, was die Folgen wären, wenn sie ihren Kollegen direkt bäte, ihr nicht mehr seine Probleme zu erzählen. In einem weiteren Schritt bringen die intervenierenden Fragen der Coach die Klientin dazu, abzuwägen, ob die beschriebenen möglichen Folgen wirklich der schlimmstmögliche Fall wären bzw. ob diese möglichen Folgen sie tatsächlich davon abhalten, gegen das unerwünschte Verhalten des Kollegen vorzugehen. Während die in Ausschnitt (4) wiedergegebene ursprüngliche Ablehnung des impliziten Vorschlags, den Kollegen zu konfrontieren, nach kurzer Verzögerung flüssig formuliert ist, zeigen die darauf folgenden Erwiderungen der Klientin Merkmale von verändertem Sprechen. Ausschnitt (9), in dem die Klientin eingesteht, sich über die Folgen einer offenen Äußerung ihres Wunschs noch keine Gedanken gemacht zu haben, enthält eine Vielzahl von Abbrüchen, Pausen und Fokusakzenten. Die Andeutung einer neuen Sichtweise in dem in (11) wiedergegebenen Ausschnitt enthält zwar zwei sich widersprechende Äußerungsteile, nach einer Verzögerung zu Beginn aber nur kürzere Pausen und am Ende der Äußerung ein schlussfolgerndes ,also inSO:fern-“. Auch wenn die Schlussfolgerung nicht ausformuliert wird, ist sie sprachlich angedeutet und stellt inhaltlich einen Perspektivwechsel zur ursprünglichen Ablehnung dar - und ist somit ein Indiz für Veränderung.

Die Coach verwendet zunächst offene, dann, zur Rückversicherung, auch geschlossene Fragen. Sie setzt zuerst das vergleichsweise neutrale Verb ,passieren ' ein, bevor sie das emotional markiertere Verb ,befürchten` zusammen mit dem negativ besetzten Superlativ ,schlimmste(n)` verwendet, was sie noch zusätzlich betont. Diese formallinguistischen Aspekte der diskutierten Fragen korrespondieren dabei funktionallinguistisch mit der hypothetischen Wirklichkeitskonstruktion des Worst Case und dessen Ratifizierung

\footnotetext{
11 Das Ausmalen eines Worst-Case-Szenarios im Coaching war, ebenso wie Fragen nach „Feenwünschen“ und andere Interventionen, Gegenstand des theoretischen Inputs in der betreffenden Weiterbildung, aus deren Kontext die Aufnahme des Coachinggesprächs stammt. Fragen i.e.S. waren hingegen erst zu einem späteren Zeitpunkt Thema.
}

und bilden damit eine interaktionsspezifische soziale Praktik im Coaching (vgl. Habscheid 2016, S. 11 ff.; Graf und Spranz-Fogasy 2018), mit der Veränderung initiiert wird.

\subsection{Veränderung "to go" auf den Punkt gebracht: den ausdruck den nehm ich auf jeden fall MIT.=}

Mit einem zweiten Fallbeispiel soll gezeigt werden, welche Bedeutung Schlüsselbegriffe haben können, um zu verstehen, was ,das Problem` (vgl. Nothdurft 1984) ist - Schlüsselbegriffe können damit zum Ausgangspunkt und zu einer Ressource (vgl. Ribeiro et al. 2013, S. 298) für Veränderung werden. Datengrundlage ist ein weiteres Kurzzeitcoaching von etwa 17 Minuten Dauer, gefolgt von einer etwa fünfminütigen Nachbesprechung. Der Weiterbildungsteilnehmer, der als Klient ein Anliegen besprechen möchte, arbeitet im Bereich der Studienberatung. Er möchte mithilfe einer Coach (und in Anwesenheit einer Beobachterin) eruieren, wie er in für ihn unerwarteten Situationen des beruflichen Alltags flexibler reagieren kann. Hierbei geht es primär um Anfragen von Kolleg/innen, die für ihre jeweiligen Studiengänge oft sehr kurzfristig sehr individuelle Beratungslösungen erbitten.

In der Nachbesprechung bewertet der Klient in einem in (13) wiedergegebenen Rückblick ein Wort als hilfreich, das die Coach verwendet hatte, um sein Problem (das nach der schließlich geteilten Einschätzung gar nicht i.e.S. sein Problem ist) auf den Punkt zu bringen:

(13) Klient: =sag mal komplexes THEma aber [dat mit dem sysTEMfehler,] ehm:: fand ich schon (.) $<<$ lachend $>$ sehr TREFfend. $>$ den ausdruck den nehm ich auf jeden fall MIT.= $(17: 42-17: 51)$

Zunächst qualifiziert er den Bereich, der ihm Probleme bereitet, als „komplexes THEma“. Der hier erwähnten Komplexität steht das einzelne Wort gegenüber, das sein Problem auf den Punkt bringt: ,sysTEMfehler“. Die positive Bewertung dieses Ausdrucks als ,sehr TREFfend“ wird von einem Lachen des Klienten begleitet. Er verstärkt seine Bewertung noch dadurch, dass er sagt ,den ausdruck den nehm ich auf jeden fall MIT“, womit der Einschätzung durch den Klienten uneingeschränkte Gültigkeit zugeschrieben wird. Ribeiro et al. (2013, S. 298) beobachten im Zusammenhang mit Therapie, dass ,formulations of the problem and strategies jointly explored [...] may turn into client resources [...] which may ultimately result in the emergence of an alternative self-narrative" (vgl. zu formulations/Reformulierungen außerdem Mack et al. 2016, S. $38 \mathrm{ff}$, $52 \mathrm{ff}$. und Scarvaglieri 2015). In diesem Zusammenhang ist interessant, dass die weitere Teilnehmerin der Weiterbildung, die das Gespräch als Beobachterin begleitet 
hat, in der Nachbesprechung hier auch von einem ,schlüsselwort" spricht. ${ }^{12}$

(14) Beobachterin: DA kam: (---) dann das schlüsselwort sysTEMfeh:ler, (.) hab ich mir auch !AHA! (--) [effekt] noTIE:RT, (19:22-19:30)

Genau wie der Klient hat sie diesen Moment als entscheidend wahrgenommen - de Haan et al. (2010, S. 113) sprechen von critical moments: „Critical moments are remembered as exciting, tense and/or significant moments after coaching conversations. They can be assumed to be a reflection of change through executive coaching as it happens in conversation." Dies soll im Folgenden mit Blick auf das eigentliche Coachinggespräch genauer aufgezeigt werden.

Zu Beginn des Kurzzeitcoachings kann der Klient seine Ziel- und Wunschvorstellung schnell und konkret benennen.

(15) Klient: ${ }^{\circ} \mathrm{hhh} \mathrm{hhh}^{\circ}(1,4)$ im grunde genommen in der LAge sei_zu sei:n (.) flexi::bler (.) auf bestimmte: $(1,9)$ unerwartete: (.) situatio::n reaGIE:ren zu können. (-) (0:05-0:17)

Die Schilderung solcher Situationen und dessen, was genau das Problem darstellt, nimmt hingegen sehr viel Zeit in Anspruch. Der Klient veranschaulicht detailreich konkrete Fälle (vgl. Scarvaglieri 2013 zu Veranschaulichungen und Graf in Vorbereitung zu Explorieren als kommunikativer Handlung) und die Coach gibt ihm Gelegenheit dazu, indem sie entsprechende Rückmeldesignale gibt, die ihre Aufmerksamkeit anzeigen, und indem sie mitunter kurze Rückfragen zu Details stellt (vgl. Albrecht und Perrin 2013, S. 37; Menzel 2008). Als sich in der Schilderung des Klienten mehr und mehr unterschiedliche Probleme vermischen, thematisiert sie dies (nicht zuletzt mit Blick auf die für diese Coachingübung zur Verfügung stehende Zeit) und klärt mit dem Klienten, dass er das ursprünglich benannte Ziel bearbeiten möchte. Sie fragt ihn nach Strategien, die er bereits ausprobiert hat, um flexibler reagieren zu können. Der Klient nimmt dies zum Anlass für weitere detaillierte Schilderungen, die sich die Coach aufmerksam anhört. Nach etwa zehn Minuten bittet sie ihn, ein Worst-Case-Szenario ${ }^{13} \mathrm{zu}$ entwerfen. Die Erwiderung des Klienten ist in (16) zitiert:

\footnotetext{
12 Der Einsatz von Schlüsselbegriffen war nicht Gegenstand des dem Kurzzeitcoaching vorangehenden theoretischen Inputs der Weiterbildung.

${ }^{13}$ Im Rahmen der Weiterbildungen stand die Intervention, dass die Klient/innen sich Worst-Case-Szenarien ausmalen, auf dem Lehrplan. Dass sie in beiden Fallbeispielen auftauchen, ist deshalb nicht unbedingt überraschend.
}

(16) Klient: worst case (.) is: im GRUNde genommen wenn:: $\ddot{o}: \mathrm{hm}:{ }^{\circ} \mathrm{hhh} \ddot{\mathrm{o}}: \mathrm{h} \mathrm{hh}^{\circ}(2,0)$ wir sind zwar ne DIENSTleistungseinrichtung (---) [!ABER!] wir können (.) nich auf alles sozusagen was aus den studiengängen a_ad hoc und EINzeln (.) eh auf uns zu:kommt: (.) können wir nicht HÄNdeln ne (.); dat ist einfach schlichtweg SO: (10:04-10:13)

Wie in (16) ersichtlich, verallgemeinert der Klient sein Problem für die Einheit, für die er arbeitet (,wir“) und benennt Grenzen (,,[!ABER!] wir können (.) nich auf alles sozusagen was aus den studiengängen a_ad hoc und EINzeln (.) eh auf uns zu:kommt: (.) können wir nicht HÄNdeln ne"). Das betonte ,[!ABER!]“ wird dabei sprachlich wieder etwas zurückgenommen, indem der Klient es durch „sozusagen“ relativiert und mittels ,ne“ gleichsam eine Bestätigung erbittet. Mit dem abschließenden ,dat is einfach schlichtweg SO:" betont er das Faktische und Entpersonalisierte und damit die Unmöglichkeit, das leisten zu können, was von ihm erwartet wird (bzw. das, was er von sich erwartet).

Nach der ausführlichen, in (16) nicht in Gänze wiedergegebenen Schilderung des Klienten fasst die Coach in einer kategorisierenden Reverbalisierung (vgl. Scarvaglieri 2015, S. 64) ihre Auffassung dessen zusammen, was das Problem ist:

(17) Coach: ((lacht)) ${ }^{\circ} \mathrm{hh}$ also: (-) is: (.) das vielleicht so die (--) des grund (.) proBLE:M (.) dass dus auch mit dir persönlich in verbindung tri_eh bit_eh beZIEH:st, (--) obwohl es eigentlich n sysTEM(.)fe:hler is:. $(11: 05-11: 15)$

(18) Klient: genau. (11:16)

Die Coach markiert ihre Zusammenfassung durch hörbares Atmen und ein langegezogenes einleitendes ,also:“. Verschiedene Pausen sowie Reformulierungen (,tri_eh bit_eh beZIEH:st“) können dahingehend interpretiert werden, dass sie unsicher ist, ob ihre Zusammenfassung das „grund (.) proBLE:M“ trifft. Ihre Erklärung des ,eigentlichen' Problems, dass es sich nämlich um einen ,sysTEM(.)fe:hler“" handelt, ist dann aber „sehr TREFfend“, wie der Klient in der bereits in (13) wiedergegebenen Äußerung aus der Nachbesprechung bestätigt. Er bestätigt dies auch schon in der in (18) zitierten unmittelbaren Erwiderung (,genau“). 
Die Coach scheint sich ihres „Treffers“ ”nicht bewusst zu sein und versucht, ihre Erklärung näher zu begründen. ${ }^{14}$

(19) Coach: also das is viellei:cht (.) ich weiß nicht was:: (--) man für strategi:en entwickeln kann: (.) daGEgenum zu sa:gen:: (.) okay es is: (.) kurzlebig es is eh::: dyna:misch und es überROLLT einen meistens; aber das: (.) liegt nicht an mir und meiner fähigkeit damit zu !AR!bei:ten. (11:17-11:33)

In (19) macht sie zunächst einen Vorstoß (,also das is viellei:cht (.)"), bricht dann aber ab und benennt ihre Ratlosigkeit (,,ich weiß nicht was:: (--) man für strategi:en entwickeln kann: (.) daGEgen-“). Daran anschließend formuliert sie - nun aus der Ich-Perspektive des Klienten - weiter und situiert das Problem nicht bei ihm (,,das: (.) liegt nicht an mir und meiner fähigkeit damit zu !AR!bei:ten“), sondern außerhalb seines direkten Einflussbereichs. Dies wird auch an einem bildhaften Ausdruck wie ,überrollen' deutlich. Indirekt referiert die Coach damit auf ,das System', benennt dieses aber nicht noch einmal explizit. Mit der Verwendung des Wortes ,dynamisch“ wiederholt sie zudem ein vom Klienten im Vorfeld eingebrachtes Wort. Der Klient greift aber nicht diese Äußerung auf, sondern die davor (vgl. (17)), wenn er sagt:

(20) Klient: es ist scho:n auch_n sysTEMfehler (.) klar; (11:34-11:36)

(21) Coach: okay:; (11:37)

Er ratifiziert damit ihre Deutung zumindest teilweise (,scho:n auch") und beschreibt sie als plausibel („klar"), was die Coach in der in (21) wiedergegebenen Äußerung wiederum mit einem „oka:y;“ ratifiziert. Anschließend lobt der Klient die Coach explizit.

(22) Klient: find ich hast du auch nochma gut zum AUSdruck gebracht. ${ }^{\circ} \mathrm{hh}$ e::h, aber man (sitzt/siehts) ja selber nich wenn man im system STECK:T::= (11:38-11:45)

Er referiert mit „AUSdruck“ auf den Schlüsselbegriff ,Systemfehler' und entschuldigt sich quasi indirekt dafür,

\footnotetext{
${ }^{14}$ Dies bestätigt sie auch in der Nachbesprechung. In gewisser Weise hat die Coach diesen Moment auch als critical moment (vgl. de Haan et al. 2010) erlebt, aber aufgrund ihrer Unsicherheit, ob ihre Intervention angemessen ist. Würde man das Kodierungsschema von de Haan et al. (2010, S. 119) anwenden, würde man den Moment aus Sicht der Coach als , ,[s]ignificant emotional experience in the moment: doubt (coach); fundamental not-knowing, often a starting point for reflection" klassifizieren, während aus Klientensicht die Kodierung ,[a] moment of learning: a moment in which new insight was created for coach and - particularly - client" treffender wäre.
}

nicht selbst darauf gekommen zu sein, dass es um ein allgemeines Problem geht, das in der Organisation und nicht in seiner Person begründet liegt. Hierfür greift er das erste Glied des Kompositums ,Systemfehler' auf und macht sich den Ausdruck damit noch mehr zu eigen (,,aber man (sitzt/ siehts) ja selber nich wenn man im system STECK:T:.="). Er porträtiert sich dabei selbst als passiv und nicht handlungsfähig (,wenn man im system STECK:T:.=“). Er kann also gar nicht, wie gewünscht oder (von sich selbst) erwartet, flexibel reagieren. ${ }^{15}$

Nach der Problemklärung und -benennung mithilfe des Schlüsselbegriffs ,Systemfehler ${ }^{\star}$ gewinnt das (hier aus Platzgründen nicht weiter wiedergegebene) Coachinggespräch deutlich an Dynamik und ist in den Formulierungen von Coach wie auch Klient klar auf Lösungen hin orientiert (vgl. Mack et al. 2016, S. $38 \mathrm{ff}$., $52 \mathrm{ff}$.). Die Coach erfragt, welche Art Anfragesituationen für den Klienten ideal wären und wie diese innerhalb der institutionellen Rahmenbedingungen (dem ,System') zu erreichen seien. Während im ersten Teil des Coachinggesprächs der Klient den größten Redeanteil hatte, steigt im zweiten Teil der Redeanteil der Coach. Die Coach beginnt sogar, selbst Lösungsmöglichkeiten zu entwickeln, stoppt sich dann aber mit einem metareflexiven Hinweis, dass dies nicht ihre Aufgabe sei. ${ }^{16}$ Sie fragt den Klienten nach Möglichkeiten der Operationalisierung der von ihm beschriebenen Idealvorstellung und vereinbart mit ihm abschließend zwei konkrete Maßnahmen, mit denen er seine Situation verbessern kann. Damit ist eine Transfermöglichkeit für die erarbeitete Veränderungsstrategie sichergestellt (vgl. Graf in Vorbereitung).

Die Coach bittet den Klienten im Anschluss daran indirekt um ein globales Feedback zum Coachinggespräch.

(23) Coach: =zuFRIEden? (17:40)

(24) Klient: ja: [bin ich] schon. (17:41-17:42)

Nach dieser mehrheitlich positiven Bewertung (die lediglich durch „schon“ eingeschränkt wird), folgt gleich die eingangs in (13) zitierte Äußerung, in der auf den Schlüsselbegriff des Systemfehlers referiert wird, der nicht nur der Schlüssel für die Problemlösung ist, sondern auch für

\footnotetext{
15 Voutilainen et al. (2011, S. 362) schildern in ihrer psychotherapeutischen Fallstudie Vergleichbares: „The patient offered material to the therapist that the therapist expressed more explicitly in her conclusion. The patient then confirmed that conclusion and then again, being again more ,on her own', in a position where she could elaborate on the therapist's formulation in her own words, the patient moved away from what was just mutually achieved. In other words, what the therapist did in making that conclusion might be seen as adopting a position that was in the patient's zone of proximal development."

16 Der Klient fragt die Coach aber in der Nachbesprechung just in dieser Beraterrolle an und möchte erfahren, welche Vorschläge sie hätte machen wollen.
} 
den Erfolg dieses kurzen Coachinggesprächs (dem, wie bereits erwähnt, im Rahmen der Weiterbildung kein Input zu Schlüsselbegriffen vorausging).

Das hier vorgestellte Beispiel zeigt, dass sich eine veränderte Sichtweise nicht nur langsam durch stetes Nachfragen entwickeln kann, sondern auch ganz plötzlich mit der Nennung eines einzelnen Wortes, das, um die Beobachterin des beschriebenen Coachings zu zitieren, einen Aha-Effekt bewirkt (vgl. (14)). Das Wort ,Systemfehler' lässt für den Klienten seine gesamte Situation plötzlich in einem anderen Licht erscheinen, als er erkennt, dass „sein“ Problem nicht i.e.S. ein Problem ist, das in seiner Person begründet liegt (oder dort zumindest nicht begründet liegen muss), sondern in äußeren Rahmenbedingungen. Deren Anpassung an das von ihm Leistbare muss (und kann) anders angegangen werden als eine Anpassung seiner Person oder Persönlichkeit. Verändertes Sprechen als Indiz für eine veränderte Sichtweise zeigt sich in diesem Beispiel nicht nur in der Wiederaufnahme des Begriffs durch den Klienten (sei es in Form des kompletten Kompositums oder des Erstglieds ,System'), sondern auch in der veränderten Dynamik und der veränderten inhaltlichen Orientierung des Gesprächs, nachdem geklärt ist, was eigentlich ,das Problem“ ist. Das Gespräch wird interaktiver und die Redeanteile von Coach und Klient gleichen sich einander quantitativ an. Es geht im Folgenden nicht mehr um ,das Problem', sondern um Strategien, wie es sich lösen lässt bzw. wie sich die Situation des Klienten (zumindest in seiner subjektiven Wahrnehmung) verbessern lässt. Hierbei gleichen sich die Beiträge von Coach und Klient auch inhaltlich an, da nun beide die gleiche Zielrichtung haben.

\section{Zusammenfassung und Ausblick}

Die vorgestellten Beispiele zeigen, wie in Coachinggesprächen Veränderungen initiiert werden können - sei es, dass durch Fragen in Verbindung mit anderen Perspektivierungsangeboten langsam eine Umwandlung oder Rekonzeptualisierung (vgl. Buchholz 2003, S. 89f.; Scarvaglieri 2015, S. $60 \mathrm{ff}$.) in Gang gesetzt wird und/oder dass ein Schlüsselbegriff plötzlich neue Interpretations- und/oder Perspektivierungsmöglichkeiten eröffnet und einem Klienten künftig als Ressource zur Problembewältigung dienen kann (vgl. Ribeiro et al. 2013, S. 298). Die mikroanalytische schrittweise Rekonstruktion der Beteiligtenperspektiven kann auf verschiedenen Ebenen des Sprachsystems (z.B. Lautung, Wortwahl oder Satzbau) zeigen, welches die (im positiven Sinne) kritischen oder innovativen Momente (vgl. de Haan et al. 2010; Ribeiro et al. 2013, S. 296f.) der Coachinggespräche sind.

Ob die in den Gesprächen sichtbaren Anzeichen von Perspektivwechseln tatsächlich zu Veränderungen im Handeln und (damit) schließlich auch zu einer Verbesserung der als belastend beschriebenen Situationen führen, kann nur über mehrere Coachingsitzungen hinweg (und/oder mithilfe zusätzlicher ethnographischer Erhebungen) beobachtet werden. Für das jeweilige Einzelgespräch ist entscheidend, dass eine veränderte Sichtweise zumindest als Ausgangspunkt für Veränderung wahrgenommen wird bzw. werden kann.

$\mathrm{Zu}$ fragen bleibt, wie und für wen Wissen über diese Möglichkeiten der Initiierung von Veränderung hilfreich sein kann. Die Arbeit mit Transkripten vermittelt Einsichten, wie Interventionen in konkreten Einzelfällen funktionieren (oder auch nicht) und wie Veränderungen angestoßen werden können. Wenn sich in großen Datenkorpora ähnliche Befunde ergeben wie in den hier vorgestellten Fallanalysen, so lassen sich daraus Good-Practice-Beispiele zum skalierenden Einsatz von Fragen sowie zum Einsatz von Schlüsselbegriffen ableiten (vgl. Abschn. 2). Diese können sowohl für bereits praktizierende Coaches als auch in Weiterbildungskontexten hilfreich sein, um ,ein schärferes Bewusstsein für [...] eigene Praktiken entwickeln [zu können], für gewisse Probleme und Schwierigkeiten im Gesprächsprozess [...] sensibilisiert [zu] werden und die Möglichkeit $[\mathrm{zu}]$ erhalten, das eigene Handlungsspektrum $\mathrm{zu}$ erweitern" (Winkler 2017, S. 3). Hierfür ist es wichtig, um die Bedeutung passender Schlüsselbegriffe (vgl. Buchholz 2003, S. 89 ff.) ebenso zu wissen wie um verschiedene Fragetechniken, ihren Einsatz, ihre Potenziale und auch ihre Grenzen in Kombination mit den je virulenten Inhalten. Hierfür wiederum ist Wissen über gesprächslinguistische Grundlagen unabdingbar.

Danksagung Dieser Beitrag basiert auf einem bei der 15. Konferenz der International Pragmatics Association in Belfast gehaltenen Vortrag. Den Verantwortlichen der Sektion „The Pragmatics of Change“, EvaMaria Graf, Claudio Scarvaglieri und Thomas Spranz-Fogasy, danke ich für die freundliche Aufnahme, und ihnen sowie allen Diskutanten für ihre Rückmeldungen. Mein besonderer Dank gilt Jan Gerwinski für hilfreiche Kommentare und Diskussionen zum Vortrag wie auch zu diesem Beitrag. Ebenso danke ich Sandra Herling und Linda Hilkenbach sowie zwei Rezensenten dieses Beitrags für ihre Kommentare, die mir viele Denkanstöße gegeben haben. Des Weiteren sei allen Teilnehmerinnen und Teilnehmern verschiedener Weiterbildungen im Bereich Hochschulcoaching und Kollegiale Beratung sowie Interkulturelles Coaching gedankt, die zugestimmt haben, dass ihre Coachinggespräche aufgenommen und in anonymisierter Form zu Forschungszwecken verwendet werden. Das Transkribieren der Daten haben - unterstützt durch die Nachwuchsförderung der Philosophischen Fakultät der Universität Siegen - Lina Azazil, Ute Reimers und Carolin Zimmermann übernommen. Auch ihnen vielen Dank!

Open Access Dieser Artikel wird unter der Creative Commons Namensnennung 4.0 International Lizenz (http://creativecommons.org/ licenses/by/4.0/deed.de) veröffentlicht, welche die Nutzung, Vervielfältigung, Bearbeitung, Verbreitung und Wiedergabe in jeglichem Medium und Format erlaubt, sofern Sie den/die ursprünglichen Autor(en) und die Quelle ordnungsgemäß nennen, einen Link zur Creative Commons Lizenz beifügen und angeben, ob Änderungen vorgenommen wurden. 


\section{Literatur}

Albrecht, C., \& Perrin, D. (2013). Zuhören im Coaching. Wiesbaden: Springer VS.

Brown, P., \& Levinson, S. (1987). Politeness. Some Universals in Language Usage. Cambridge: Cambridge University Press.

Buchholz, M.B. (2003). Relationen und Konvergenzen - Neue Perspektiven der Psychoanalyse. Psychotherapeutenjournal, 2, 87-96.

Buchholz, M.B., \& Reich, U. (2015). Tanz der Einsicht - Linguistische Einblicke in ein psychoanalytisches Gespräch. Journal für Psychologie, 23(2), 11-51.

Graf, E.-M. (2007). "I'm not embarrassed" - "A little ... uncomfortable? Is that nearer?" The negotiation of meaning in the discourse of coaching. In P. Cap \& J. Nijakowska (Hrsg.), Current Trends in Pragmatics (S. 281-302). Cambridge: Cambridge Scholar Press.

Graf, E.-M. (2015). Kommunikative Basisaktivitäten im CoachingGespräch: Ein linguistischer Beitrag zur Coaching-Prozessforschung. Coaching | Theorie \& Praxis. https://doi.org/10.1365/ s40896-015-0001-x.

Graf, E.-M., \& Pawelczyk, J. (2014). The interactional accomplishment of feelings-talk in psychotherapy and executive coaching: same format, different functions? In E.-M. Graf, M. Sator \& T. Spranz-Fogasy (Hrsg.), Discourses of Helping Professions (S. 59-89). Amsterdam, Philadelphia: Benjamins.

Graf, E.-M., \& Spranz-Fogasy, T. (2018). Welche Frage, wann und warum? Eine qualitativ-linguistische Programmatik zur Erforschung von Frage-Sequenzen als zentrale Veränderungspraktik im Coaching. Coaching I Theorie \& Praxis. https://doi.org/10. 1365/s40896-018-0021-4

Graf, E.-M., Aksu, Y., \& Rettinger, S. (2010). Qualitativ-diskursanalytische Erforschung von Coaching-Gesprächen. Organisationsberatung - Supervision - Coaching, 17, 133-149.

Graf, E.-M., Sator, M., \& Spranz-Fogasy, T. (2014). Discourses of helping professions: contexts and contextualization. In E.-M. Graf, M. Sator \& T. Spranz-Fogasy (Hrsg.), Discourses of Helping Professions (S. 1-12). Amsterdam, Philadelphia: Benjamins.

Graf, E.-M. (im Erscheinen). The Discourses of Executive Coaching. Linguistic Insights into Emotionally Intelligent Coaching. Amsterdam, Philadelphia: Benjamins.

de Haan, E., Bertie, C., Day, A., \& Sills, C. (2010). Critical moments of clients and coaches: a direct-comparison study. International Coaching Psychology Review, 5(2), 109-128.

Habscheid, S. (2015). Beratung, Coaching, Supervision: Formen helfender Interaktion in Unternehmen. In M. Hundt \& D. Biadala (Hrsg.), Handbuch Sprache in der Wirtschaft (S. 256-271). Berlin, Boston: de Gruyter.

Habscheid, S. (2016). Handeln in Praxis. Hinter- und Untergründe situierter sprachlicher Bedeutungskonstitution. In A. Deppermann, H. Feilke \& A. Linke (Hrsg.), Sprachliche und Kommunikative Praktiken (S. 127-151). Berlin, Boston: de Gruyter.

Jautz, S. (2017). Immer auf Augenhöhe? Ein Blick in den sprachlichen Werkzeugkoffer im Coaching. In M. Dräger \& M. Kuhnhenn (Hrsg.), Sprache in Rede, Gespräch und Kommunikation. Linguistisches Wissen in Weiterbildungen zur Kommunikationskompetenz (S. 47-64). Frankfurt am Main: Peter Lang.

Kallmeyer, W. (2000). Beraten und Betreuen. Zur gesprächsanalytischen Untersuchung von helfenden Interaktionen. Zeitschrift für Qualitative Bildungs-, Beratungs- und Sozialforschung, 2, 227-252.
Konerding, K.-P. (2015). Heilung durch Sprache und Sprechen - Linguistik und Psychotherapie. In A. Busch \& T. Spranz-Fogasy (Hrsg.), Handbuch Sprache in der Medizin (S. 225-242). Berlin, Boston: Walter de Gruyter.

Leiman, M., \& Stiles, W.B. (2001). Dialogical sequence analysis and the zone of proximal development as conceptual enhancements to the assimilation model: the case of Jan revisited. Psychotherapy Research, 11, 311-330.

Mack, C., Nikendei, C., Ehrenthal, J.C., \& Spranz-Fogasy, T. (2016). „[...] hab ich glaub ich die richtigen fragen gestellt“. Therapeutische Fragehandlungen in psychodiagnostischen Gesprächen. OPAL - Online Publizierte Arbeiten zur Linguistik, 3, 1-98.

Menzel, K. (2008). Zuhören für Fortgeschrittene. Eine kommunikationswissenschaftliche Analyse, guten Zuhörens' und gesprächspsychotherapeutischer Kommunikation. Duisburg: Universitätsverlag Rhein-Ruhr.

Nazarkiewicz, K., \& Krämer, G. (2012). Handbuch Interkulturelles Coaching. Konzepte, Methoden, Kompetenzen kulturreflexiver Begleitung. Göttingen: Vandenhoeck \& Ruprecht.

Nothdurft, W. (1984). ,....̈h folgendes problem äh... “ Die interaktive Ausarbeitung ,des Problems“ in Beratungsgesprächen. Tübingen: Narr.

Nothdurft, W., Reitemeier, U., \& Schröder, P. (Hrsg.). (1994). Beratungsgespräche: Analyse asymmetrischer Dialoge. Tübingen: Narr.

Ribeiro, E., Ribeiro, A.P., Gonçalves, M. M., Horvath, A. O., \& Stiles, W. B. (2013). How collaboration in therapy becomes therapeutic: the therapeutic collaboration coding system. Psychology and Psychotherapy, 86(3), 294-314.

Scarvaglieri, C. (2013). Sprachliches Veranschaulichen und kuratives Verstehen in der Psychotherapie. In K. Birkner \& O. Ehmer (Hrsg.), Veranschaulichungsverfahren im Gespräch (S. 66-92). Mannheim: Verlag für Gesprächsforschung.

Scarvaglieri, C. (2015). Reverbalisierungen als Brücke zum kollektiven Handlungswissen: Eine gesprächsanalytische Untersuchung therapeutischer Interventionen. Journal für Psychologie, 23(2), 53-80.

Selting, M., Auer, P., Barth-Weingarten, D., Bergmann, J., Bergmann, P., Birkner, K., Couper-Kuhlen, E., Deppermann, A., Gilles, P., Günthner, S., Hartung, M., Kern, F., Mertzlufft, C., Meyer, C., Morek, M., Oberzaucher, F., Peters, J., Quasthoff, U., Schütte, W., Stukenbrock, A., \& Uhmann, S. (2009). Gesprächsanalytisches Transkriptionssystem 2 (GAT2). Gesprächsforschung - OnlineZeitschrift zur verbalen Interaktion, 10, 353-402. http://www. gespraechsforschung-ozs.de.

Spranz-Fogasy, T. (2014). Anticipatory reactions. Patients' answers to doctors' questions. In E.-M. Graf, M. Sator \& T. Spranz-Fogasy (Hrsg.), Discourses of Helping Professions (S. 205-226). Amsterdam, Philadelphia: Benjamins.

Voutilainen, L., Peräkylä, A., \& Ruusuvuori, J. (2011). Therapeutic change in interaction: conversation analysis of a transforming sequence. Psychotherapy Research, 21(3), 348-365.

Wehrle, M. (2011). Die 100 besten Coaching-Übungen. Bonn: managerSeminare.

Wehrle, M. (2012). Die 500 besten Coaching-Fragen. Das große Workbook für Einsteiger und Profis zur Entwicklung der eigenen Coaching-Fähigkeiten. Bonn: managerSeminare.

Winkler, O. (2017). Anwendung der Gesprächsanalyse als FeedbackInstrument im Chat-Coaching. Coaching I Theorie \& Praxis. https://doi.org/10.1365/s40896-017-0016-6. 